\title{
An analytic approach to modeling the optical response of anisotropic nanoparticle arrays at surfaces and interfaces
}

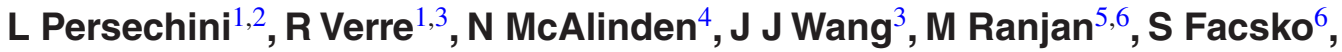 \\ I V Shvets ${ }^{1,3}$ and J F McGilp ${ }^{1}$ \\ ${ }^{1}$ School of Physics, Trinity College Dublin, Dublin 2, Ireland \\ 2 Centre for Industrial and Engineering Optics, School of Physics, Dublin Institute of Technology, \\ Kevin Street, Dublin 8, Ireland \\ ${ }^{3}$ Centre for Research on Adaptive Nanostructures and Nanodevices (CRANN), Trinity College Dublin, \\ Dublin 2, Ireland \\ ${ }^{4}$ Institute of Photonics, SUPA, University of Strathclyde, Glasgow G4 0NW, UK \\ ${ }^{5}$ FCIPT, Institute for Plasma Research, Gandhinagar, India \\ ${ }^{6}$ Institute of Ion Beam Physics and Materials Research, Helmholtz-Zentrum Dresden-Rossendorf, \\ 01314 Dresden, Germany \\ E-mail: jmcgilp@tcd.ie
}

Received 9 January 2014, revised 19 February 2014

Accepted for publication 27 February 2014

Published 20 March 2014

\begin{abstract}
Anisotropic nanoparticle (NP) arrays with useful optical properties, such as localized plasmon resonances (LPRs), can be grown by self-assembly on substrates. However, these systems often have significant dispersion in NP dimensions and distribution, which makes a numerical approach to modeling the LPRs very difficult. An improved analytic approach to this problem is discussed in detail and applied successfully to NP arrays from three systems that differ in NP metal, shape and distribution, and in substrate and capping layer. The materials and anisotropic NP structures that will produce LPRs in desired spectral regions can be determined using this approach.
\end{abstract}

Keywords: plasmonics, optical response, anisotropic, arrays, modeling

(Some figures may appear in colour only in the online journal)

\section{Introduction}

Metallic nanoparticles (NPs) have excitation modes that are highly localized and decay rapidly within the structures, resulting in localized plasmon resonances (LPRs) [1, 2]. These excitation modes have been the subject of considerable interest due to their potential application in areas such as biological sensors [3-6], enhancement effects [7-9] and solar cell applications [10-12]. One of the key aspects of LPRs is the possibility of tuning the resonance position by altering the NP size, shape and aspect ratio, by modifying the NP distribution in, or on, the host material, and by varying the host material itself [13].

Recently, polarization-dependent optical methods have been demonstrated to be a very useful tool for both characterization of LPRs and for practical applications. Phase sensitive methods, such as spectroscopic ellipsometry (SE) [14], have been demonstrated to have a striking advantage in sensitivity [15, 16], up to single molecule recognition [17]. Another polarization method, reflectance anisotropy spectroscopy (RAS), has been used to monitor the evolution of NP arrays growing on facetted $\alpha-\mathrm{Al}_{2} \mathrm{O}_{3}$ surfaces by self-assembly [18-20]: structures with LPRs covering the whole visible range can be produced using this approach [21].

NP arrays grown on planar surfaces by lithographic techniques typically have periodic structures of NPs with high symmetry, which allows the use of numerical solutions of Maxwell's equations to determine the optical response, such as boundary element [22] and finite difference time domain methods [23]. The anisotropic NP arrays grown on surfaces 
by self-assembly, however, have significant dispersion in NP dimensions and distribution, which makes a numerical approach very difficult. Analytic solutions exist for regular arrays of spheroidal NPs supported on substrates, where the rotational axis of the NP is normal to the surface [24], and these have been used to model polarized absorption spectra $[25,26]$. Exact analytic solutions have not been published for the ellipsoidal, or flattened ellipsoidal, NPs that typically grow by self-assembly.

A basic analytic approach for estimating the optical response of such anisotropic systems has been outlined that provides reasonable agreement with experiment for $\mathrm{Ag}$ NP arrays grown by glancing angle deposition onto facetted $\alpha-\mathrm{Al}_{2} \mathrm{O}_{3}$ under high vacuum conditions $[16,20]$. Here an improvement to this approach is discussed in detail and applied successfully to NP arrays from three systems that differ in NP metal, shape and distribution, and also in substrate and in capping layer. Anisotropic NPs typical of selfassembled growth, anisotropic arrays grown on structured substrates, tarnish shells, and single or multiple capping layers can all be modeled, using only the thickness and the mixing fraction for the effective medium of the NP layer as free parameters to be optimized for particular material systems. The materials and anisotropic NP structures that will produce LPRs in desired spectral regions can be determined using this improved analytic approach.

\section{Experiment}

The experimental procedures used for the growth and characterization of the three distinct plasmonic systems have been reported in detail previously [18, 27, 28]. In the first system, anisotropic $\mathrm{Pb}$ nanostructures were grown on clean $\mathrm{Si}(5 \mathrm{5}$ 7) surfaces by self-assembly under ultra-high vacuum (UHV) conditions [27]. Gold and $\mathrm{Pb}$ were deposited from molecular beam epitaxy cells, and $a$-Si was evaporated using a sublimation source. Deposition of 0.18 monolayers (ML) of $\mathrm{Au}$ onto the vicinal $\mathrm{Si}$ surface produces aligned, single height atomic steps, which act as a template for the growth of highly anisotropic $\mathrm{Pb}$ islands [29]. Deposition of $\mathrm{Au}$ and $\mathrm{Pb}$ was monitored optically using a visible/near-IR $(0.45-5 \mathrm{eV})$ RAS system, which uses photoelastic modulation (PEM) to change the polarization state of the light [30]. This technique has been shown to be a sensitive probe of aligned, anisotropic phases grown on vicinal Si surfaces [31-33]. RAS measures the difference in reflectance, at near normal incidence, of light linearly polarized in two orthogonal directions at the surface plane of a cubic material [34]:

$$
\frac{\Delta r}{r}=2 \frac{r_{x}-r_{y}}{r_{x}+r_{y}}
$$

where $r_{x}$ and $r_{y}$ are the complex Fresnel reflection coefficients for the surface for light polarized in the $x$ and $y$ directions, with the steps and long axis of the $\mathrm{Pb}$ islands running in the $x$ direction. For the $\mathrm{Si} / \mathrm{Pb}$ system a rotating sample RAS system was used to extend the spectral range of the RAS measurements down to $0.12 \mathrm{eV}$ in the ambient, using a Coherent Ti : sapphire-regenerative amplifier-optical parametric amplifier-DFG system that produced linearly polarized 130 fs pulses, with a $100 \mathrm{kHz}$ repetition rate, tuneable from 0.12 to $0.5 \mathrm{eV}$ in the IR [27]. In situ optical measurements were repeated after the $\mathrm{Pb}$ nanostructures had been capped with $4.5 \mathrm{~nm}$ of $a$-Si.

Anisotropic Ag nanostructures were grown by selfassembly using two different techniques on two different substrates. Glancing angle deposition onto facetted $\alpha-\mathrm{Al}_{2} \mathrm{O}_{3}$ under high vacuum conditions, using a collimated Ag flux, produced arrays of anisotropic Ag NPs decorating the facets [18]. Growth was monitored in situ using the PEMbased RAS. The second technique used was the deposition of $\mathrm{Ag}$ onto native-oxide-covered rippled $\mathrm{Si}\left(\begin{array}{lll}0 & 0 & 1\end{array}\right)$ substrates under high vacuum conditions. The substrates were produced at the Helmholtz-Zentrum Dresden-Rossendorf using a procedure that has been described in detail previously $[35,36]$. Briefly, the rippled structure with $\sim 30 \mathrm{~nm}$ periodicity and $\sim 1 \mathrm{~nm}$ amplitude, measured by atomic force microscopy (AFM), were prepared by irradiating native-oxide-covered $\mathrm{Si}\left(\begin{array}{lll}0 & 0 & 1\end{array}\right)$ substrates at room temperature with a collimated beam of $500 \mathrm{eV} \mathrm{Ar}^{+}$ions, aligned with the $<100>$ azimuth and at an angle of $67^{\circ}$ with respect to the surface normal. Fluences in the range $\sim 10^{16}-\sim 10^{19}$ ions $\mathrm{cm}^{-2}$ were used [37]. After ex situ characterization, Ag was deposited perpendicularly to the ripples on the native-oxide-covered surface by electron beam evaporation at an incidence angle of $70^{\circ}$, followed by sample annealing at $350^{\circ} \mathrm{C}$. RAS and SE were used to monitor the linear optical response in the ambient for both types of Ag nanostructures.

\section{Theory}

Analytic solutions for NP systems exist for spheroidal particles, including truncated caps, on surfaces or embedded at interfaces, but none exist where a rotational axis normal to the surface or interface is absent [24]. An approximate analytic theory is developed here that provides a reasonable description of the plasmonic resonances of these three very different anisotropic NP systems. The procedure involves determining the anisotropic polarizability of an isolated ellipsoidal NP, then estimating the dielectric response of the NP layer, and finally calculating the optical response of the multilayer system. This approximate analytic approach for anisotropic systems provides reasonable agreement with experiment and thus may be used to guide the choice of materials and growth parameters for the fabrication of aligned anisotropic NP arrays for producing LPRs in particular spectral regions.

\subsection{The isolated NP}

The dielectric function of the metal NP, some or all of whose dimensions are shorter than the electron inelastic scattering mean free path in the bulk metal, is modified in direction $j$ to account for the increased scattering rates from the boundaries of the ellipsoidal NP [38] and becomes anisotropic: 
Table 1. Material parameters for bulk $\mathrm{Ag}$ and $\mathrm{Pb}$.

\begin{tabular}{llll}
\hline & $\omega_{\mathrm{p}}(\mathrm{eV})$ & $\gamma_{\mathrm{b}}(\mathrm{eV})$ & $\nu_{\mathrm{F}}\left(\mathrm{m} \mathrm{s}^{-1}\right)$ \\
\hline $\mathrm{Ag}$ & $8.85[48]$ & $0.037[48]$ & $1.4 \times 10^{6}[46]$ \\
$\mathrm{Pb}$ & 8.65 & 0.20 & $2.96 \times 10^{6}[47]$ \\
\hline
\end{tabular}

$$
\begin{aligned}
\varepsilon_{j}(\omega) & =\varepsilon_{\text {bulk }}(\omega)+\frac{\omega_{\mathrm{p}}^{2}}{\omega\left(\omega+\mathrm{i} \gamma_{\mathrm{b}}\right)}-\frac{\omega_{\mathrm{p}}^{2}}{\omega\left(\omega+\mathrm{i} \gamma_{j}\right)} \text { and } \\
\omega_{\mathrm{p}} & =\left(\frac{n e^{2}}{\varepsilon_{0} m^{*}}\right)^{1 / 2}
\end{aligned}
$$

where $\varepsilon_{\text {bulk }}(\omega)$ is the experimental bulk metal dielectric function, $\gamma_{\mathrm{b}}$ is the bulk scattering rate, $n=z \rho N_{\mathrm{A}} / M$ is the number density of conduction electrons (where $z$ is the valence, $\rho$ is the density, $N_{\mathrm{A}}$ is Avogadro's number and $M$ is the molecular weight), and $m^{*}$ is the plasmon effective mass [39]. The form of equation (2) arises from taking the experimental bulk dielectric function, which includes interband transitions, then removing the bulk free-electron-like response and replacing it with a modified free-electron-like response appropriate to NPs. The modified scattering rate

$$
\gamma_{j}=\gamma_{\mathrm{b}}+\frac{A v_{\mathrm{F}}}{R_{j}}
$$

where $v_{\mathrm{F}}$ is the Fermi velocity of the conduction electrons, $R_{j}$ is the semi-axis of the ellipsoid and $A$ is generally treated as a fitting parameter typically varying between $\sim 0.2$ and $\sim 2$ [40-42]. The increase in scattering rate leads to homogeneous broadening of the plasmon resonance of a single NP. Material parameters for $\mathrm{Ag}$ and $\mathrm{Pb}$ are given in table 1 and it can be seen that the bulk broadening in $\mathrm{Pb}$ is almost an order of magnitude larger than that of $\mathrm{Ag}$. The $\mathrm{Pb}$ values were estimated by fitting a Drude model to consolidated visible and IR dielectric function data [43-45]. Fermi velocities vary with crystal orientation but Ag has a nearly-free-electron-like Fermi surface. The deviation from the free electron value is less than $10 \%$ [46], allowing the free electron value to be used; for $\mathrm{Pb}$, the value in the $\langle 111\rangle$ direction was chosen [47].

A theoretical treatment of the $A$ parameter for Ag identified a free NP size-dependent term and a NP interface term that depends on the surrounding medium and adsorbates [49]. The interface contribution varies with capping layer dielectric function, and the contribution from the perpendicular component of the interface term was shown to be smaller. Values in the middle of the calculated range were chosen and used for both $\mathrm{Ag}$ and $\mathrm{Pb}$ NPs. The three scattering rates in equation (2), in $\mathrm{eV}$, become

$$
\begin{aligned}
& \gamma_{x}=\gamma_{\mathrm{b}}+0.65\left(\frac{\hbar}{e}\right) \frac{\nu_{\mathrm{F}}}{R_{x}}, \quad \gamma_{y}=\gamma_{\mathrm{b}}+0.65\left(\frac{\hbar}{e}\right) \frac{v_{\mathrm{F}}}{R_{y}}, \\
& \gamma_{z}=\gamma_{\mathrm{b}}+0.4\left(\frac{\hbar}{e}\right) \frac{v_{\mathrm{F}}}{R_{z}} .
\end{aligned}
$$

Using these expressions removes $A$ as a variable and appears to produce reasonable lineshapes.

\subsection{The NP in a dielectric environment}

When the NP is embedded in an environment with a dielectric function of $\varepsilon_{\text {cap }}$, the field experienced by the NP is modified by the local field, which differs from the external applied field. The anisotropic polarizability of an ellipsoid of volume $V=4 \pi R_{x} R_{y} R_{z} / 3$ is

$$
\alpha_{j}=V \frac{\varepsilon_{j}-\varepsilon_{\text {cap }}}{\varepsilon_{\text {cap }}+L_{j}\left(\varepsilon_{j}-\varepsilon_{\text {cap }}\right)}
$$

where $L_{j}$ is the shape depolarization factor for ellipsoidal particles, accounting for the local field experienced by a single $\mathrm{NP}$, and $\varepsilon_{j}$ is the anisotropic dielectric function of the metal NP introduced above [1]. The quasi-static approximation is used in obtaining equation (5), which is an adequate approach for NP systems where no multipolar modes or multiple scattering effects are observed. The modified long wavelength approximation can be used for larger particles to take into account retardation effects [50].

The standard depolarization factors for isolated ellipsoidal NPs are expressed as

$$
\begin{aligned}
L_{j} & =\frac{j k l}{2} \int_{0}^{\infty} \frac{\mathrm{d} q}{\left(j^{2}+q\right) \sqrt{\left(j^{2}+q\right)\left(k^{2}+q\right)\left(l^{2}+q\right)}}, \\
\sum L_{j} & =1
\end{aligned}
$$

where $j, k, l$ are the semi-axes of the ellipsoid and the sum rule follows from evaluating the integral [51]. The effect of anisotropic local fields is to split the resonance into three components.

Modeling the response becomes much more difficult for NPs assembled in a single layer at the interface between the substrate and ambient, or substrate and capping layer, as an image charge now alters the local field significantly. For a flattened ellipsoid with the center a distance $d$ from the interface in the $z$-direction, the depolarization factor becomes $[26,52]$

$$
\begin{aligned}
\bar{L}_{x, y} & =L_{x, y}-\frac{1}{24} F \frac{R_{x} R_{y} R_{z}}{d^{3}} \\
\bar{L}_{z} & =L_{z}-\frac{1}{12} F \frac{R_{x} R_{y} R_{z}}{d^{3}}
\end{aligned}
$$

where $F$ is the image charge scaling factor. The sum rule in equation (6) now breaks down and the depolarization factors sum to less than unity [24]. For a sphere in the vicinity of a substrate, $F=\left(\varepsilon_{\mathrm{b}}-\varepsilon_{\text {cap }}\right) /\left(\varepsilon_{\mathrm{b}}+\varepsilon_{\text {cap }}\right)$, where $\varepsilon_{\mathrm{b}}$ is the bulk dielectric function of the substrate and $\varepsilon_{\text {cap }}$ is the dielectric function of the medium surrounding the sphere $\left(\varepsilon_{\text {cap }}=1\right.$ for uncapped samples) [53]. However, interfacial NP layers are heterogeneous and may be rough, suggesting an effective medium dielectric function, $\varepsilon_{\text {eff }}$, should be used. There are well known formulae [54], such as Maxwell-Garnett and Bruggeman, for spherical inclusions, which can be adapted for ellipsoidal NPs. Different approaches were explored, but the fundamental bounds to $\varepsilon_{\text {eff }}$ [54] limited the shifts in resonance energies to $\sim 0.2 \mathrm{eV}$. As the interfacial NP layer is generally not well defined, and the shifts are small, a simple arithmetic model was adopted: $F=\left(\varepsilon_{\mathrm{b}}-\varepsilon_{\text {eff }}\right) /\left(\varepsilon_{\mathrm{b}}+\varepsilon_{\text {eff }}\right)$, where $\varepsilon_{\text {eff }}=(1-f) \varepsilon_{\mathrm{b}}+f \varepsilon_{\text {cap }}$. 
The mixing fraction $f$ is allowed to vary from system to system to optimize agreement with experiment. For consistency, $\varepsilon_{\text {cap }}$ is replaced by $\varepsilon_{\text {eff }}$ in equation (5).

These expressions allow a particle to be located above the interface, but they also provide a simple way of adjusting the strength of the image charge interaction by varying $d$. The standard spherical dipole approach, which places $d$ at the center of the NP, is known to overestimate the interaction with the substrate. This affects the LPR response of all components, but particularly that of the $z$-component $[24,26]$. An analytic solution is available for flattened NPs with rotational symmetry about the surface normal [24, chapter 6], which provides a corrected value for $d$. The ellipsoidal NPs are approximated as oblate spheroidal particles with the geometric average inplane radius [19], which allows an effective value of $d$ to be determined from the analytic expressions:

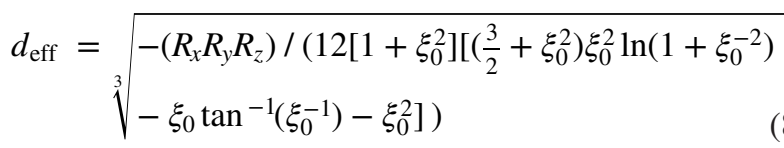

where

$$
\xi_{0}=R_{z} / \sqrt{R_{x} R_{y}-R_{z}^{2}} .
$$

This provides a consistent way of adjusting $d$ although, as the anisotropy of the NP increases, $d_{\text {eff }}$ becomes unphysical.

Oxidation or tarnishing on exposing metallic NPs to the ambient is a common occurrence and the effect can be approximated by assuming that the NP is coated by a dielectric shell. The polarizability for a shell of thickness $d_{\mathrm{sh}}$ and dielectric function $\varepsilon_{\mathrm{sh}}$ becomes [51, 55]:

$$
\begin{aligned}
\alpha_{j}=V \frac{\left(\varepsilon_{\mathrm{sh}}-\varepsilon_{\mathrm{eff}}\right)\left[\varepsilon_{\mathrm{sh}}+\left(\varepsilon_{j}-\varepsilon_{\mathrm{sh}}\right)\left(1-f_{v}\right) L_{j}\right]+f_{v} \varepsilon_{\mathrm{sh}}\left(\varepsilon_{j}-\varepsilon_{\mathrm{sh}}\right)}{\left(\left[\varepsilon_{\mathrm{sh}}+\left(\varepsilon_{j}-\varepsilon_{\mathrm{sh}}\right)\left(1-f_{v}\right) L_{j}\right]\left[\varepsilon_{\mathrm{eff}}+\left(\varepsilon_{\mathrm{sh}}-\varepsilon_{\mathrm{eff}}\right) L_{j}\right]\right.} \\
\left.\quad+f_{v} \varepsilon_{\mathrm{sh}}\left(\varepsilon_{j}-\varepsilon_{\mathrm{sh}}\right) L_{j}\right)
\end{aligned}
$$

where the core volume fraction of the NP is approximated by

$$
f_{v}=\left(\sqrt{R_{x} R_{y}} / \sqrt{R_{x} R_{y}}+d_{\mathrm{sh}}\right)^{3} .
$$

Where there is no shell, $d_{\mathrm{sh}}=0$ and $\varepsilon_{\mathrm{sh}}=\varepsilon_{\text {eff }}$, regaining equation (5).

\subsection{The NP layer}

The electromagnetic coupling between the NPs has to be considered when the NPs are assembled in a single layer at an interface. Within a point dipole coupling model, the layer dielectric functions are $[25,52,56]$

$$
\begin{gathered}
\varepsilon_{x, y}^{L}=\varepsilon_{\mathrm{eff}}\left(1+\frac{\rho \alpha_{x, y}}{1+\varepsilon_{0} \varepsilon_{\mathrm{eff}} \alpha_{x, y} \beta_{x, y}}\right) \\
\frac{1}{\varepsilon_{z}^{L}}=\frac{1}{\varepsilon_{\mathrm{eff}}}\left(1-\frac{\rho \alpha_{z}}{1+\varepsilon_{0} \varepsilon_{\mathrm{eff}} \alpha_{z} \beta_{z}}\right)
\end{gathered}
$$

where $\rho$ is the volume density. The volume density becomes the surface density for the single layer NP systems considered here. The interaction parameters arising from the dipolar coupling are

$$
\begin{aligned}
\beta_{x, y}= & -\frac{1}{4 \pi \varepsilon_{0} \varepsilon_{\mathrm{eff}}}\left(F \frac{1}{(2 d)^{3}}+\sum_{i} \frac{3 \cos ^{2} \theta_{i}-1}{r_{i}^{3}}\right. \\
& \left.+F \sum_{i} \frac{3 \cos ^{2} \theta_{i}^{\prime}-1}{r_{i}^{\prime}}\right) \\
\beta_{z}= & -\frac{1}{4 \pi \varepsilon_{0} \varepsilon_{\mathrm{eff}}}\left(F \frac{2}{(2 d)^{3}}+\sum_{i} \frac{3 \cos ^{2} \theta_{i}-1}{r_{i}^{3}}\right. \\
& \left.+F \sum_{i} \frac{3 \cos ^{2} \theta_{i}^{\prime}-1}{r_{i}^{\prime 3}}\right)
\end{aligned}
$$

where $\theta_{i}$ is the dipole angle, $r_{i}$ is the separation of the point dipole from the central particle, $\theta_{i}^{\prime}$ is the image dipole angle, $r_{i}^{\prime}$ is the separation of the image point dipole from the central particle. The first term is the self-image interaction, the second term is the dipole interaction, and the third term is the image dipole interaction. The NPs are assumed to form a rectangular lattice, with a center-to-center spacing of $l_{x}$ and $l_{y}\left(\rho=1 / l_{x} l_{y}\right.$ and the average NP layer thickness is $\rho V$ ). Summing over a $20 \times 20$ lattice is sufficient to reduce the finite sum error to less than $5 \%$. In addition, the contribution at large separations is overestimated in equations $(11 a)$ and $(11 b)$ because retardation, which reduces the dipolar contribution due to decoherence, is neglected.

\subsection{The optical response of the multilayer system}

The effective dielectric function of the NP layer is treated as one layer of the full optical model of the multilayer system, which uses the transfer matrix approach. This was originally formulated for isotropic media [57], but has been extended by Berreman [58] and by Schubert $[59,60]$ to produce a more general form applicable to anisotropic layers. Following this approach, a four-vector is defined for the s- and p-polarized components of the electric and magnetic field, and each layer can be then defined by a $4 \times 4$ partial transfer matrix, $T_{\mathrm{p}}$. Knowing the thickness and dielectric properties of each layer allows the partial transfer matrix of each individual layer to be calculated and the full response of the system derived in the form of complex reflection coefficients, which can be compared with experiment. In outline,

$$
T_{\mathrm{p}}=\exp (\mathrm{i} \omega d \Delta / c)=\beta_{0} I+\beta_{1} \Delta+\beta_{2} \Delta^{2}+\beta_{3} \Delta^{3}
$$

where $d=\rho V$, the average NP layer thickness and the complex $\beta_{i}$ must obey the following set of equations:

$$
\exp \left(\mathrm{i} \omega d q_{k} / c\right)=\sum_{j=0}^{3} \beta_{j} q_{k}^{j}, \quad k=1, \ldots, 4
$$

where $q_{k}$ are the eigenvalues of $\Delta$ (appendix) [60]. The total matrix is obtained by multiplying the inverse of the incident matrix, $L_{a}$, the partial transfer matrices, $T_{\mathrm{p} i}$, of the $N$ layers, and the exit matrix, $L_{f}$ : 
Table 2. Parameters for $\mathrm{Si}(55$ 7)-5 × 1-Au/8 ML Pb, and Si (5 5 7)$5 \times 1-\mathrm{Au} / 8 \mathrm{ML} \mathrm{Pb} / 4.5 \mathrm{~nm} a$-Si. The range of experimental values was determined from SEM images.

\begin{tabular}{lll}
\hline & Experiment & Model \\
\hline$R_{x}(\mathrm{~nm})$ & $85-215$ & 110 \\
$R_{y}(\mathrm{~nm})$ & $30-90$ & 35 \\
$R_{z}(\mathrm{~nm})$ & $6[61]$ & 6.5 \\
$l_{x}(\mathrm{~nm})$ & $360-620$ & 390 \\
$l_{y}(\mathrm{~nm})$ & $110-230$ & 140 \\
Island density $\rho=1 / l_{x} l_{y}\left(\mu m^{-2}\right)$ & $7-25$ & 18 \\
Average NP layer thickness $(\mathrm{nm})$ & $2.3(2)$ & 1.9 \\
RAS scaling factor & - & 0.13 \\
Capping layer thickness $(\mathrm{nm})$ & 4.5 & 4.5 \\
Effective medium $f$ & - & 0.5 \\
\hline
\end{tabular}

$$
T=L_{a}^{-1} \prod_{i=1}^{N} T_{\mathrm{p} i}\left(-d_{i}\right) L_{f} .
$$

The incident and exit matrices are given in the appendix. The complex reflection coefficients required are found from the components of $\boldsymbol{T}$ :

$$
\left.\begin{array}{l}
r_{s s}=\left(T_{21} T_{33}-T_{23} T_{31}\right) / \Delta T \\
r_{p p}=\left(T_{11} T_{43}-T_{13} T_{41}\right) / \Delta T
\end{array}\right\} \quad \text { where } \quad \Delta T=T_{11} T_{33}-T_{13} T_{31} .
$$

Finally, the measured quantities are expressed in terms of the reflection coefficients. For RAS,

$$
\frac{\Delta r}{r}=2 \frac{r_{p p}-r_{s s}}{r_{p p}+r_{s s}}
$$

with normally the real part of $\Delta r / r$ being measured. For SE, it is the complex pseudo-dielectric function, $\left\langle\varepsilon_{i}\right\rangle, i=x, y$, that can be determined directly from measurements in the $x z$ and yz optical plane:

$$
\left\langle\varepsilon_{i}\right\rangle=\sin ^{2} \Theta+\sin ^{2} \Theta \tan ^{2} \Theta\left(\frac{T_{21} T_{33}-T_{11} T_{43}}{T_{21} T_{33}+T_{11} T_{43}}\right)^{2}
$$

where $\Theta$ is the angle of incidence and the principle axes of the layers are aligned with the laboratory axes [59]. This approach can be simply extended to multiple capping layers.

The dielectric functions of the NP material, substrate and capping layer are known, leaving seven variable parameters, which were adjusted manually: the NP dimensions and spacing, the mixing fraction for the effective medium of the NP layer, and a RAS scaling factor chosen to reproduce the experimental $x$ resonance peak height. The last factor is included as RAS is only directly sensitive to anisotropic NPs, while the experimental NP surface density will generally count all the NPs.

\section{Results and discussion}

\subsection{LPRs of Pb islands grown on Si (5 5 7)- $5 \times 1-A u$}

Analysis of the SEM images of the 8 ML coverage reveals islands of typical width $60 \mathrm{~nm}$, with some coalescing into islands of double or triple that width. The length distribution was from 170 to $430 \mathrm{~nm}$. Previous work has indicated
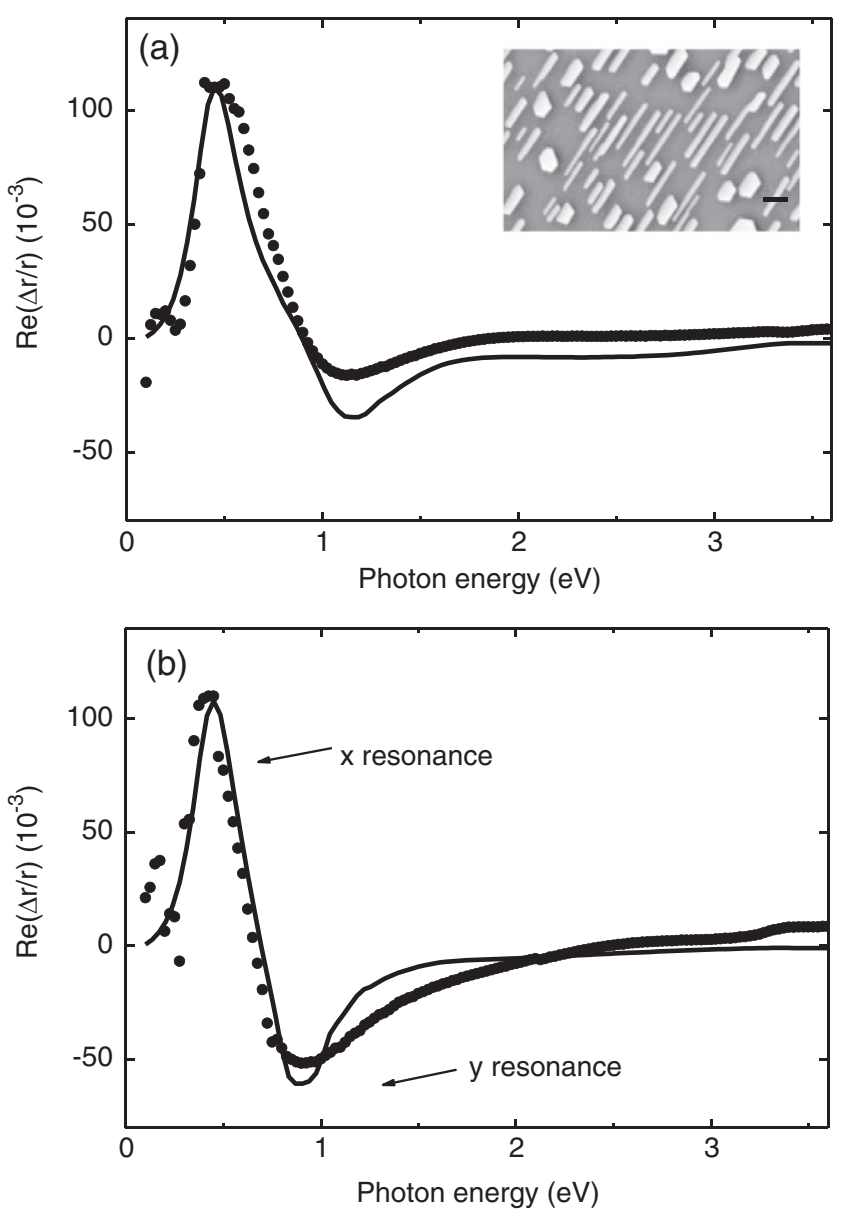

Figure 1. RAS spectra of $8 \mathrm{ML} \mathrm{Pb}$ deposited on $\mathrm{Si}(557)-5 \times 1$ $\mathrm{Au}$. Data points and model spectra (solid line) (a) uncapped; the inset shows an SEM image with a $200 \mathrm{~nm}$ scale bar; $(b)$ capped with $4.5 \mathrm{~nm}$ of $a$-Si.

that the height of the islands is relatively uniform at $12 \mathrm{~nm}$ in this coverage region [61]. For $8 \mathrm{ML} \mathrm{Pb}, 30 \%$ of the surface was covered in islands. Table 2 records the large variation in island size, shape and distribution, as this is more useful than average values. Figure 1(a) shows the real RAS response from uncapped $\operatorname{Si}(55$ 7)-5×1-Au/8 ML Pb, where the inset shows a typical SEM image. The noisier data below $0.45 \mathrm{eV}$ were recorded with the rotating sample laser RAS assembly. Figure $1(b)$ is the response of the same sample capped with $4.5 \mathrm{~nm}$ of $a-\mathrm{Si}$, as measured using a quartz crystal oscillator. These experimental data have been reported previously [27], where a simple antenna model [62] produced reasonable agreement with the $x$-LPR position, but the model does not address the other resonances, nor the line shape and intensity.

Two large structures can be seen in the IR region of the uncapped spectrum: a minimum at $1.13 \mathrm{eV}$ ( $y$-LPR) and a maximum at $0.47 \mathrm{eV}(x$-LPR). RAS is a normal incidence technique and does not probe the $z$-LPR. The laser RAS, while quite noisy, confirms the presence and position of the maximum, which lies outside the spectral range of conventional RAS instruments. Capping then red-shifts the peaks to $0.9 \mathrm{eV}$ and $0.42 \mathrm{eV}$, respectively, reduces their width, and increases the relative contribution of the $y$-LPR. The 

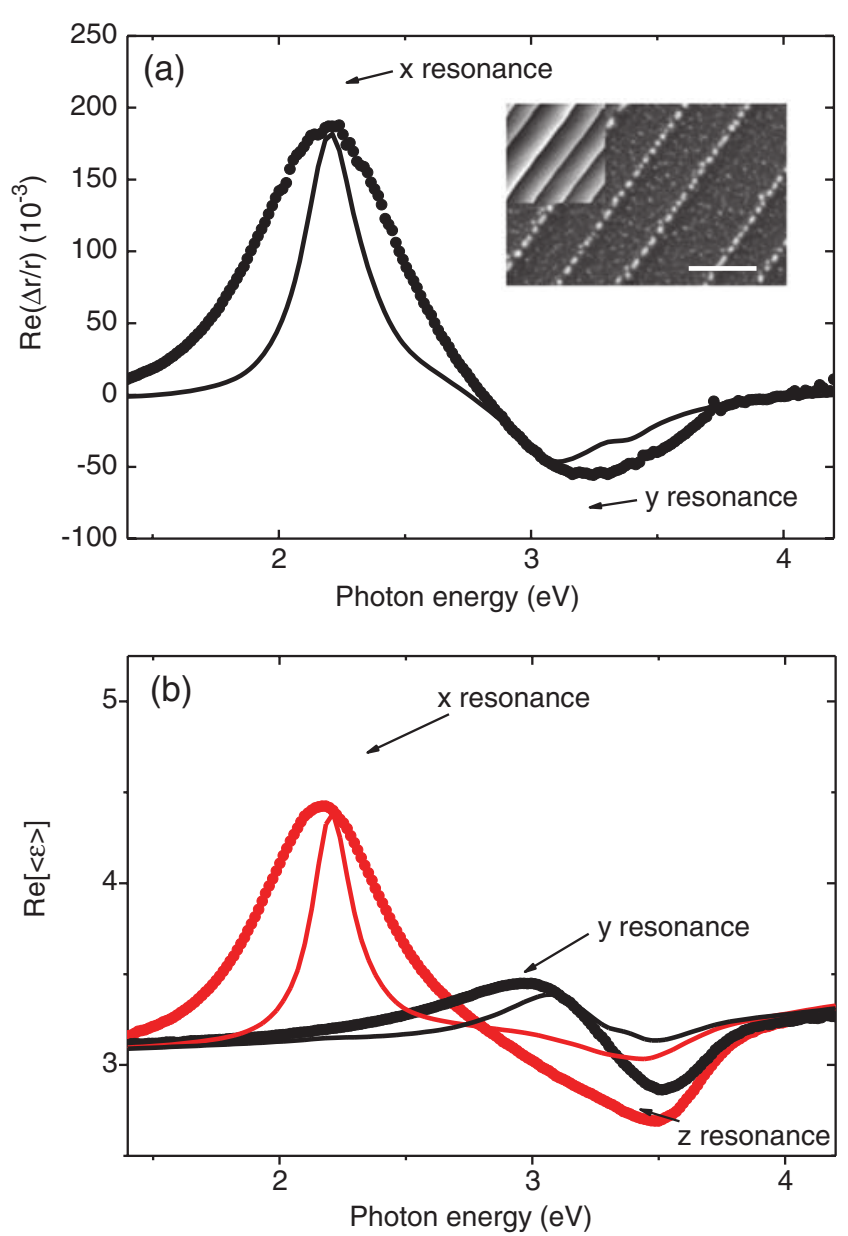

Figure 2. Spectra of Ag islands grown on facetted $\alpha-\mathrm{Al}_{2} \mathrm{O}_{3}$ : data points and model (solid line) (a) RAS; the inset shows an SEM image of the bare facetted substrate (left), and the substrate with the NP array, with a $200 \mathrm{~nm}$ scale bar; $(b)$ real part of the pseudo-dielectric function determined from SE measurements in the $x z$-plane (red) and the $y z$-plane (black), with model spectra (solid lines)

experimental data are compared with the model parameters in table 2. The same model parameters are used for both capped and uncapped samples, the only change being the addition of the capping layer of $a$-Si. The dielectric function of the $a$-Si was determined from SE measurements of a similar $a$-Si layer grown under the same conditions on native-oxide-covered $\mathrm{Si}\left(\begin{array}{lll}0 & 0 & 1\end{array}\right)$. The model captures the position and shape of both spectra satisfactorily, using parameter values that are consistent with the experimental data. The peak width is dominated by the bulk plasmon contribution, because the inelastic scattering length of $\sim 10 \mathrm{~nm}$ is much smaller than the island dimensions. The effective medium chosen was the average of the substrate and vacuum, or substrate and capping layer, as appropriate. Reasonable results are obtained when modeling the uncapped and capped spectra using a single parameter set, which is a quite severe test of the model. No additional structure indicative of quadrupolar contributions can be seen. The overall agreement is impressive, particularly when it is clear from the inset in figure 1 $(a)$ that the NP shapes are facetted and only very approximately ellipsoidal.
Table 3. Parameters for Ag NPs grown on facetted $\alpha-\mathrm{Al}_{2} \mathrm{O}_{3}$. Estimated errors are given in parentheses.

\begin{tabular}{lll}
\hline & Experiment [63] & Model \\
\hline$R_{x}(\mathrm{~nm})$ & $9.3(2.3)$ & 10.0 \\
$R_{y}(\mathrm{~nm})$ & $7.7(1.4)$ & 6.5 \\
$R_{z}(\mathrm{~nm})$ & - & 5.0 \\
$l_{x}(\mathrm{~nm})$ & $24(6)$ & 22 \\
$l_{y}(\mathrm{~nm})$ & $120(35)$ & 130 \\
Island density $\rho=1 / l_{x} l_{y}\left(\mu m^{-2}\right)$ & $350(130)$ & 350 \\
Average NP layer thickness $(\mathrm{nm})$ & $3.5(5)$ & 0.5 \\
RAS scaling factor & - & 1.0 \\
Effective medium $f$ & - & 0.66 \\
Tarnish shell thickness $d_{\mathrm{sh}}(\mathrm{nm})$ & - & 0.3 \\
\hline
\end{tabular}

\subsection{LPRs of Ag islands grown on facetted $\alpha-\mathrm{Al}_{2} \mathrm{O}_{3}$}

These experimental results have been reported previously, where a simpler version of the analytic model was used [63]. The arbitrary scaling factor that was applied to the dipolar interaction is eliminated in the approach presented here. Figure 2(a) shows the real RAS response and figure $2(b)$ the real part of the pseudo-dielectric function in the $x z-$ and $y z-$ optical planes determined by SE, all spectra being measured in the ambient. The dielectric function of $\mathrm{Ag}_{2} \mathrm{~S}$ tarnish films was used for the dielectric shell in equation (9a) [64]. The tarnish grows slowly and a shell thickness of $0.3 \mathrm{~nm}$ was chosen, consistent with previous work [55], which produced a small red shift of $<0.1 \mathrm{eV}$ in the spectra. The proportions of the effective medium used were one-third substrate and twothirds ambient. The RAS and SE data were modeled using the same parameters.

The experimental data are compared with the model parameters in table 3. The average values of the Ag NP size, shape and distribution, with error estimates, for the arrays were obtained from the SEM images. The much smaller, isotropic NPs on the terraces are omitted from the analysis, as discussed in previous work [18, 63]. The average NP layer thickness in the model is thus smaller than the experimentally estimated thickness of the deposited layer. There is good agreement with the RAS line shape in figure 2(a), with the positions and relative intensities of the LPRs being well reproduced. The experimental peak widths are broader than the model, however, due to the dispersion in NP size. The peak width is dominated by the NP geometry for this system, because the Ag bulk plasmon inelastic scattering length of $\sim 25 \mathrm{~nm}$ is larger than the island dimensions. No significant quadrupolar structure is observed, in agreement with previous work, which showed that tarnishing of Ag NPs suppressed quadrupolar effects [55]. The model SE response in figure $2(b)$ shows the same general characteristics as the RAS for the $x$ - and $y$-LPRs, but the model predicts a smaller $z$-LPR intensity than is observed. This probably reflects the limitation of the simple approach used to account for the overestimate of the dipolar interaction normal to the surface, as discussed in section 3.2. In contrast to the RAS measurements, the smaller isotropic NPs on the terraces should contribute to the overall SE response, but no additional features are observed, probably due to the smearing out of the resonances due to NP size dispersion. 

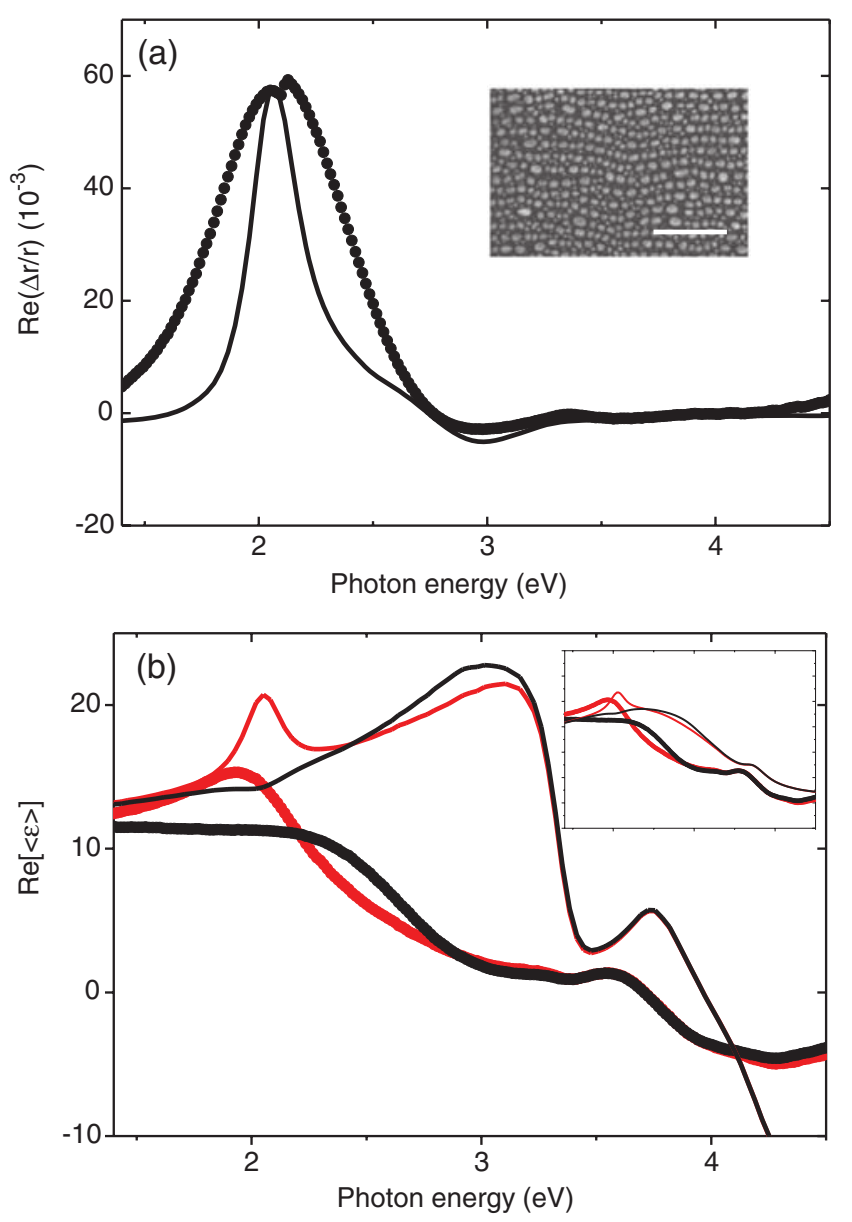

Figure 3. Spectra of Ag islands grown on native-oxide-covered rippled $\mathrm{Si}\left(\begin{array}{l}0 \\ 0\end{array}\right.$ 1): data points and model (solid line) (a) RAS; the inset shows an SEM image with a $200 \mathrm{~nm}$ scale bar; $(b)$ real part of the pseudo-dielectric function determined from SE measurements in the $x z$-plane (red) and the $y z$-plane (black), with model spectra (solid lines); the inset uses a mixed $a-\mathrm{Si} / \mathrm{SiO}_{2}$ substrate dielectric function.

\subsection{LPRs of Ag islands grown on native-oxide-covered rippled $\mathrm{Si}\left(\begin{array}{lll}0 & 0 & 1\end{array}\right)$}

The inset in figure 3(a) shows that the NPs are less well ordered than the previous examples, with the NPs assembling in wavy lines on the surface. Figure 3(a) shows the real RAS response and figure $3(b)$ the real part of the pseudo-dielectric function in the $x z$ - and $y z$-optical planes determined by SE, all spectra being measured in the ambient. The dielectric function of a native-oxide-covered rippled $\operatorname{Si}\left(\begin{array}{lll}0 & 0 & 1\end{array}\right)$ substrate, produced under the same conditions, was determined independently using SE measurements and was found to be optically isotropic. The tarnish shell parameters from the previous section were used, and the RAS and SE data were modeled with the same parameters (table 4). The two major differences in modeling this rippled sample were that, firstly, good agreement could only be obtained by using the ambient dielectric function as the effective medium, rather than a combination of substrate and ambient or capping layer dielectric functions and, secondly, the substrate contribution to the SE response in figure $3(b)$ in the direct optical gap region of $\sim 3 \mathrm{eV}$ is clearly wrong. The inset in the

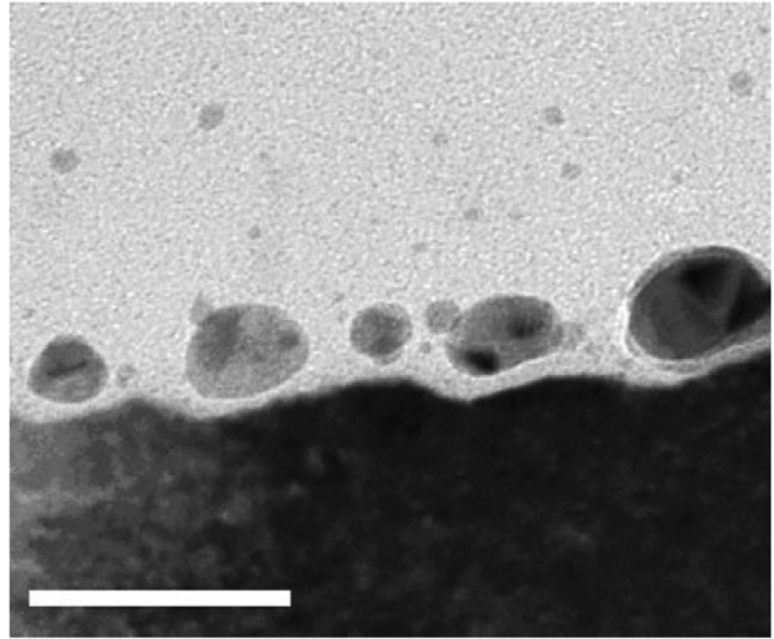

Figure 4. Cross-sectional TEM image of Ag NPs on a native-oxidecovered rippled silicon substrate, with a $50 \mathrm{~nm}$ scale bar. The larger particles are located in the ripple valleys (after [65]).

figure shows the effect of using an average of $a$-Si and $\mathrm{SiO}_{2}$ for the substrate response, which produces better agreement but is unphysical, merely being used to illustrate the substrate problem. Figure 4 shows a typical TEM cross-section of such samples, where the separation of the NPs from the rippled substrate by the $\mathrm{SiO}_{2}$ native layer is clearly seen [65]. A more complete model would need to include the effect of the native oxide, but this is not straightforward for rippled substrates.

The experimental data are compared with the model parameters in table 4. The average values of the Ag NP size, shape and distribution, with error estimates, for the arrays were obtained from the SEM images. As with the previous system, there is good agreement with the RAS line shape in figure $3(a)$, with the positions and relative intensities of the LPRs being well reproduced, and the experimental peak widths being broader than the model due to the dispersion in NP size. No significant quadrupolar structure is observed. For RAS, the simple approach of neglecting the native oxide contribution works sufficiently well, probably because RAS suppresses the isotropic substrate contribution, and the ambient and native oxide dielectric functions are similar. For SE, it is clear from figure $3(b)$ that the substrate needs to be better modeled to allow useful LPR parameters to be extracted. The advantage of using RAS for more complex substrates is clear, although this sacrifices information about the $z$-LPR.

\section{Discussion}

The analytic model produces very good agreement with experiment for $x$ - and $y$-LPRs from a range of NP materials, shapes and distributions, from different substrates, and for samples with and without capping layers. The NP dimensions range over an order of magnitude, while the NP density ranges over two orders of magnitude. The modeled RAS response, which is only sensitive to the $x$ - and $y$-LPRs and which also suppresses the substrate response, agrees very well with all the 
Table 4. Parameters for Ag NPs grown on native-oxide-covered rippled $\operatorname{Si}\left(\begin{array}{lll}0 & 0 & 1\end{array}\right)$. Estimated errors are given in parentheses.

\begin{tabular}{lll}
\hline & Experiment & Model \\
\hline$R_{x}(\mathrm{~nm})$ & $8.5(2.5)$ & 9.6 \\
$R_{y}(\mathrm{~nm})$ & $6.5(1.5)$ & 6.0 \\
$R_{z}(\mathrm{~nm})$ & - & 2.8 \\
$l_{x}(\mathrm{~nm})$ & $25(5)$ & 23 \\
$l_{y}(\mathrm{~nm})$ & $28(6)$ & 26 \\
Island density $\rho=1 / l_{x} l_{y}\left(\mu m^{-2}\right)$ & $1400(400)$ & 1672 \\
Average NP layer thickness $(\mathrm{nm})$ & - & 1.1 \\
RAS scaling factor & - & 0.4 \\
Effective medium $f$ & - & 1.0 \\
Tarnish shell thickness $d_{\text {sh }}(\mathrm{nm})$ & - & 0.3 \\
\hline
\end{tabular}

experimental data. For the Ag NPs, the width of the LPRs is dominated by the particle geometry, because the bulk scattering length is significantly larger than the NP dimensions. NP dispersion then produces a broadened experimental line shape relative to the model. For this type of system it may be possible to estimate the NP dispersion from the RAS results by using a range of NP dimensions, but this is not attempted here.

The SE results probe, in addition, the $z$-LPR, but the substrate response is now significant. This is less important for large optical gap substrates like $\mathrm{Al}_{2} \mathrm{O}_{3}$, where all three LPRs are easily identified, but appears to become problematic for semiconductor substrates, where the $z$-LPR, which is at the highest energy due to the oblate nature of NP ellipsoids grown on substrates, merges into the substrate response. The $z$-LPR modeling is generally less successful, which probably reflects the limitation of the simple approach used to account for the overestimate of the dipolar interaction normal to the surface, as discussed in section 3.2. In addition, the transfer matrix approach assumes sharp interfaces and an effective dielectric function for the NP layer was introduced to deal with both the roughness and inhomogeneity of this interfacial region. For applications only employing $x$ - and $y$-LPRs, RAS offers a simpler approach to modeling and characterization. This was particularly evident in the more complex rippled $\operatorname{Si}\left(\begin{array}{lll}0 & 0 & 1\end{array}\right)$ sample, where modeling the substrate response will be difficult but appears to be essential in producing reasonable agreement with the SE measurements.

\section{Conclusion}

An improved analytic approach to modeling LPRs has been discussed in detail and applied successfully to anisotropic NP arrays grown by self-assembly on substrates. The polarized optical response of three systems that differ in NP metal, shape and distribution, in substrate, and in capping layer was simulated and compared with RAS and SE measurements. The agreement obtained indicates that this analytic approach should be useful in determining the materials and anisotropic NP structures that will produce LPRs in desired spectral regions. Finally, the RAS response has been shown to be simpler to model than the SE response for arrays grown on semiconducting substrates, allowing the LPR information to be more easily extracted.

\section{Acknowledgments}

This work has been funded by Science Foundation of Ireland, contract nos 06/IN.1/I91 and 11/RFP.1/PHY/3047, the Irish Research Council for Science, Engineering and Technology (IRCSET), and conducted under the framework of the INSPIRE programme, funded by the Irish Government's Programme for Research in Third Level Institutions, Cycle 4, National Development Plan 2007-2013. Funding from the Deutsche Forschungsgemeinschaft (DFG FOR 845) is gratefully acknowledged.

\section{Appendix. $\Delta, L_{a}$ and $L_{f}$ matrices}

For the systems investigated, the principle axes of the layers are aligned with the laboratory axes, the incident beam is in the ambient or vacuum, and the angle of incidence is $\Theta$. The capping layer and NP layer $\Delta$ matrix becomes

$$
\Delta=\left[\begin{array}{cccc}
0 & 0 & 0 & 1-\left(\sin ^{2} \Theta / \varepsilon_{z}\right) \\
0 & 0 & -1 & 0 \\
0 & -\varepsilon_{y}+\sin ^{2} \Theta & 0 & 0 \\
\varepsilon_{x} & 0 & 0 & 0
\end{array}\right] .
$$

The capping layer was isotropic in the systems studied: $\varepsilon_{x}=\varepsilon_{y}=\varepsilon_{z}=\varepsilon_{\text {cap. }}$. The NP layer is anisotropic: $\varepsilon_{x}=\varepsilon_{x}^{L} ; \varepsilon_{y}=\varepsilon_{y}^{L} ; \varepsilon_{z}=\varepsilon_{z}^{L}$; with $\varepsilon^{L}$ given by equations $(10 a)$ and $(10 b)$. The inverse of the incident matrix, and the matrix describing exit into the substrate, are [59]

$$
L_{a}^{-1}=\left[\begin{array}{cccc}
0 & \frac{1}{2} & -\frac{1}{2} \sec \Theta & 0 \\
0 & \frac{1}{2} & \frac{1}{2} \sec \Theta & 0 \\
\frac{1}{2} \sec \Theta & 0 & 0 & \frac{1}{2} \\
-\frac{1}{2} \sec \Theta & 0 & 0 & \frac{1}{2}
\end{array}\right]
$$

$$
L_{f}=\left[\begin{array}{cccc}
0 & 0 & \sqrt{1-\left(\sin ^{2} \Theta / \varepsilon_{\mathrm{b}}\right)} & 0 \\
1 & 0 & 0 & 0 \\
-\sqrt{\varepsilon_{\mathrm{b}}-\sin ^{2} \Theta} & 0 & 0 & 0 \\
0 & 0 & \sqrt{\varepsilon_{\mathrm{b}}} & 0
\end{array}\right] .
$$

\section{References}

[1] Kreibig U and Vollmer M 1995 Optical Properties of Metal Clusters (New York: Springer)

[2] Brongersma M L and Kik P G 2007 Surface Plasmon Nanophotonics (Berlin: Springer)

[3] Willets K A and Van Duyne R P 2007 Annu. Rev. Phys. Chem. 58267

[4] Homola J 2008 Chem. Rev. 108462

[5] Hao F, Sonnefraud Y, Dorpe P V, Maier S A, Halas N J and Nordlander P 2008 Nano Lett. 83983

[6] Verellen N, Van Dorpe P, Huang C, Lodewijks K, Vandenbosch G A E, Lagae L and Moshchalkov V V Nano Lett. 11391 
[7] Nie S and Emory S R 1997 Science 2751102

[8] Kim S, Jin J, Kim Y-J, Park I-Y, Kim Y and Kim S-W 2008 Nature $\mathbf{4 5 3} 757$

[9] Kinkhabwala A, Yu Z, Fan S, Avlasevich Y, Mullen K and Moerner W E 2009 Nature Photon. 3654

[10] Atwater H A and Polman A 2010 Nature Mater. 9205

[11] Pala R A, White J, Barnard E, Liu J and Brongersma M L 2009 Adv. Mater. 213504

[12] Munday J N and Atwater H A Nano Lett. 112195

[13] Kelly K L, Coronado E, Zhao L L and Schatz G C 2003 J. Phys. Chem. B 107668

[14] Azzam R and Bashara N 1977 Ellipsometry and Polarized Light (Amsterdam: North-Holland)

[15] Losurdo M et al 2009 J. Nanopart. Res. 111521

[16] Verre R, Modreanu M, Ualibek O, Fox D, Fleischer K, Smith C, Zhang H, Pemble M, McGilp J F and Shvets I V 2013 Phys. Rev. B 87235428

[17] Kravets V G et al 2013 Nature Mater. 12304

[18] Verre R, Fleischer K, Sofin R G S, McAlinden N, McGilp J F and Shvets I V 2011 Phys. Rev. B 83125432

[19] Verre R, Fleischer K, McGilp J F, Fox D, Behan G, Zhang H and Shvets I V 2012 Nanotechnology 23035606

[20] Ualibek O, Verre R, Bulfin B, Usov V, Fleischer K, McGilp J F and Shvets I V 2013 Nanoscale 54923

[21] Verre R, Fleischer K, Ualibek O and Shvets I V 2012 Appl. Phys. Lett. 100031102

[22] Garcia de Abajo F J and Howie A 2002 Phys. Rev. B 65 115418

[23] Girard C and Dereux A 1996 Rep. Prog. Phys. 59657

[24] Bedeaux D and Vlieger J 2004 Optical Properties of Surfaces (London: Imperial College Press)

[25] Camelio S, Babonneau D, Lantiat D, Simonot L and Pailloux F 2009 Phys. Rev. B 80155434

[26] Valamanesh M, Borensztein Y, Langlois C and Lacaze E 2011 J. Phys. Chem. C 1152914

[27] McAlinden N, Wang J J and McGilp J F 2012 Phys. Status Solidi $b \mathbf{2 4 9} 1105$

[28] Persechini L, Ranjan M, Grossmann F, Facsko S and McGilp J F 2012 Phys. Status Solidi b 2491173

[29] Jalochowski M and Bauer E 2001 Surf. Sci. 480109

[30] Fleischer K, Carroll L, Smith C and McGilp J F 2007 J. Phys.: Condens. Matter 19266003

[31] McGilp J F 2001 Phys. Status Solidi a 1881361

[32] Chandola S, Hinrichs K, Gensch M, Esser N, Wippermann S, Schmidt W G, Bechstedt F, Fleischer K and McGilp J F 2009 Phys. Rev. Lett. 102226805

[33] McAlinden N and McGilp J F 2010 Europhys. Lett. 9267008

[34] Weightman P, Martin D S, Cole R J and Farrell T 2005 Rep. Prog. Phys. 681251

[35] Ranjan M and Facsko S 2012 Nanotechnology 23485307
[36] Ranjan M, Facsko S, Fritzsche H and Mukherjee S 2013 Microelectron. Eng. 102

[37] Keller A, Rossbach S, Facsko S and Möller W 2008 Nanotechnology 19135303

[38] David E 1939 Z. Phys. 114389

[39] Borensztein Y, Alameh R and Roy M 1994 Phys. Rev. B 501973

[40] Kreibig U and Fragstein C V 1969 Z. Phys. A 224307

[41] Granqvist C G and Hunderi O 1977 Phys. Rev. B 163513

[42] Alvarez M M, Khoury J T, Schaaff T G, Shafigullin M N, Vezmar I and Whetten R L 1997 J. Phys. Chem. B 1013706

[43] Mathewson A G and Myers H P 1971 Phys. Scr. 4291

[44] Ordal M A, Long L L, Bell R J, Bell S E, Alexander R W Jr, Ward C A and Bell R R 1983 Appl. Opt. 221099

[45] Ordal M A, Bell R J, Alexander R W, Long L L Jr and Querry M R 1987 Appl. Opt. 26744

[46] Mitchell J W and Goodrich R G 1985 Phys. Rev. B 324969

[47] Lykken G I, Geiger A L, Dy K S and Mitchell E N 1971 Phys. Rev. B 41523

[48] Flaten C J and Stern E A 1975 Phys. Rev. B 11638

[49] Pinchuk A, Kreibig U and Hilger A 2004 Surf. Sci. 557269

[50] Yang W-H, Schatz G C and Van Duyne R P 1995 J. Chem. Phys. 103869

[51] Bohren C F and Huffman D R 1983 Absorption and Scattering of Light by Small Particles (New York: Wiley)

[52] Yamaguchi T, Yoshida S and Kinbara A 1974 Thin Solid Films 21173

[53] Pinchuk A, Hilger A, Von Plessen G and Kreibig U 2004 Nanotechnology 151890

[54] Esquivel-Sirvent R and Schatz G C 2011 Phys. Rev. A 83042512

[55] Flores-Camacho J M, Sun L D, Saucedo-Zeni N, Weidlinger G, Hohage M and Zeppenfeld P 2008 Phys. Rev. B 78075416

[56] Yamaguchi T, Yoshida S and Kinbara A 1973 Thin Solid Films 1863

[57] Born M and Wolf E 1999 Principles of Optics (Cambridge: Cambridge University Press)

[58] Berreman D W 1972 J. Opt. Soc. Am. 62502

[59] Schubert M 1996 Phys. Rev. B 534265

[60] Schubert M, Tiwald T E and Woollam J A 1999 Appl. Opt. 38177

[61] Jalochowski M, Strozak M and Zdyb R 2003 Appl. Surf. Sci. 211209

[62] Novotny L 2007 Phys. Rev. Lett. 98266802

[63] Verre R, Fleischer K, Smith C, McAlinden N, McGilp J F and Shvets I V 2011 Phys. Rev. B 84085440

[64] Bennett J M, Stanford J L and Ashley E J 1970 J. Opt. Soc. Am. $\mathbf{6 0} 224$

[65] Oates T H, Keller A, Facsko S and Mücklich A 2007 Plasmonics 247 